\title{
Erratum to: Effects of acute relapses on neuropsychological status in multiple sclerosis patients
}

\author{
S. A. Morrow $\cdot$ S. Jurgensen $\cdot$ F. Forrestal $\cdot$ \\ Frederick E. Munchauer $\cdot$ R. H. B. Benedict
}

Published online: 2 April 2011

(C) Springer-Verlag 2011

\section{Erratum to: J Neurol}

DOI 10.1007/s00415-011-5975-3

Table 1 was published with errors. Correct table is given here:

The online version of the original article can be found under doi:10.1007/s00415-011-5975-3.

S. A. Morrow - R. H. B. Benedict

Jacobs Neurological Institute,

State University of New York at Buffalo,

Buffalo, NY, USA

S. Jurgensen - F. Forrestal - F. E. Munchauer

Biogen Idec, Inc., Cambridge, MA, USA

R. H. B. Benedict $(\bowtie)$

Buffalo General Hospital, 100 High Street,

Buffalo, NY 14203, USA

e-mail: benedict@buffalo.edu
Table 1 Pre-relapse demographics and disability measures

\begin{tabular}{|c|c|c|c|}
\hline & Cases & Controls & $p$ value \\
\hline \multicolumn{4}{|l|}{ Age (years) } \\
\hline Mean \pm SD & $44.0 \pm 7.1$ & $42.3 \pm 7.2$ & $0.387 *$ \\
\hline \multicolumn{4}{|l|}{ Gender } \\
\hline$N(\%)$ female & $35(66.0 \%)$ & $75(65.2 \%)$ & $0.917^{+}$ \\
\hline \multicolumn{4}{|c|}{ EDSS prior to relapse } \\
\hline Median (range) & $3.0(1.0-7.5)$ & $2.5(0.0-7.0)$ & $0.717^{+}$ \\
\hline \multicolumn{4}{|c|}{ Cerebral FS prior to relapse } \\
\hline Median (range) & $0.0(0.0-2.0)$ & $0.0(0.0-2.0)$ & $0.777^{+}$ \\
\hline \multicolumn{4}{|c|}{ SDMT 2 visits prior to relapse } \\
\hline Mean \pm SD & $52.3 \pm 11.6$ & $52.5 \pm 11.3$ & $0.937 *$ \\
\hline \multicolumn{4}{|c|}{ SDMT 1 visit prior to relapse } \\
\hline Mean \pm SD & $53.6 \pm 10.9$ & $53.4 \pm 11.6$ & $0.658 *$ \\
\hline \multicolumn{4}{|c|}{ MSNQ 1 visit prior to relapse } \\
\hline Mean \pm SD & $15.0 \pm 11.2$ & $13.0 \pm 10.8$ & $0.112 *$ \\
\hline \multicolumn{4}{|c|}{ MSNQ 2 visits prior to relapse } \\
\hline Mean \pm SD & $14.1 \pm 10.6$ & $13.3 \pm 11.0$ & $0.666^{*}$ \\
\hline \multicolumn{4}{|c|}{ Corticosteroid treatment } \\
\hline$N(\%)$ & $37(56.9 \%)$ & N/A & \\
\hline
\end{tabular}

